\title{
QAM Synthesis by Direct Modulation of Semiconductor Lasers under Injection Locking
}

\author{
R. Slavík ${ }^{(1)}$, J. Kakande ${ }^{(1,2)}$, R. Phelan ${ }^{(3)}$, J. O'Carroll ${ }^{(3)}$, B.Kelly ${ }^{(3)}$, and D.J. Richardson ${ }^{(1)}$ \\ 1. ORC, University of Southampton, SO17 1BJ, Southampton, UK \\ 2. Bell Labs, Alcatel-Lucent, Holmdel, NJ 07733, USA \\ 3. Eblana Photonics, Dublin, Ireland
}

\begin{abstract}
A new scheme for direct synthesis of QAM $(4,16,64, .$.$) from binary RF data streams is$ suggested and tested by generating 10 Gbaud QPSK. Current semiconductor lasers should allow for operation up to 160 Gbaud.

OCIS codes: (060.2340) Fiber optics components; (060.2380) Fiber optics sources and detectors
\end{abstract}

\section{Introduction}

Coherent optical communications allows for better resilience to transmission-related linear and nonlinear impairments (e.g., using binary phase shift keying, BPSK) and/or for increased capacity via the transmission of several bits per symbol (e.g., QPSK, 16 QAM and higher). For these modulation formats, which exploit modulation of phase, direct modulation of the signaling laser is generally not possible due to the high chirp associated with such a modulation approach. Instead, an external IQ modulator is typically used, made from materials such as $\mathrm{LiNbO}_{3}$ or InP. Although this approach clearly works well it brings several drawbacks: (i) a relatively high cost; (ii) significant insertion loss and limited optical power handling; (iii) the need for high drive voltage RF booster amplifiers; and (iv), a typical IQ modulator requires the processing (division into I and Q) and associated multiplexing of multiple high-speed binary electronic data signals, resulting in increased power loss and power consumption, noise and nonlinearity (which is particularly detrimental when the I and Q data streams have more than two levels, e.g., for 16QAM, each has 4 levels). This last feature can be mitigated by using more complicated modulators with multiple electrical inputs and multiplexing in the optical domain [1], however, this comes at the cost of a higher optical insertion loss which in turn compromises the achievable OSNR (a critical issue when working with advanced modulation format signals).

Here, we propose a new solution that simultaneously avoids all four issues associated with the use of external IQ modulators. It is based on ultralow chirp direct modulation of injection-locked (IL) semiconductor lasers followed by coherent superposition and coherent carrier suppression of their output. We show experimental results for BPSK and QPSK modulation, although it is straightforward to extend the basic concept to 16 QAM and higher modulation formats. We note that several techniques have previously been reported for constant-envelope modulation (e.g. BPSK, QPSK) based on direct laser or SOA based phase modulation [2][3][4], with some of these incorporating injection-locking [4]. However, in our opinion, these schemes cannot be straightforwardly extended to higher modulation formats (e.g. 16 QAM), offer limited performance and/or are applicable only to RZ formats.

\section{Principle of operation}

For simplicity, let us consider the generation of BPSK modulation signals first. QPSK and higher modulation formats will also be discussed shortly. Fig. 1a shows the operating principle. We first injection-lock a directly modulated laser by feeding a component of $\mathrm{CW}$ light from a master laser into a current-modulated slave laser. If both lasers emit at the same wavelength, they become phase locked, meaning that the slave frequency follows that of the master, even in the presence of modulation of the slave laser current. This is an established method to suppress the chirp of directly-modulated lasers [5], enabling low-chirp amplitude modulation of the slave laser. Moreover, as the slave laser output is phase-locked to the master, stable interference between the master laser and the modulated slave is possible. Consequently, the carrier of the amplitude modulated slave laser can be eliminated by interfering it destructively with an appropriate portion of the CW master laser light, as shown in Fig. 1a. This produces BPSK modulation.

The key features of this technique are (i) the slave produces ultralow-chirp amplitude modulation, and (ii) the slave is phase locked to the CW master thereby allowing for carrier removal. This can be simply extended to QPSK, see Fig. 1b. Here, we modulate two lasers with two binary data streams, both of them injection-locked to the same master. As the two modulated lasers are phase locked, we can combine them with a 90 degree optical phase shift, producing a four-level modulation that resembles QPSK, except for the fact that it contains the carrier. Similarly to the way BPSK signals can be synthesized, the carrier is suppressed through destructive interference with a CW 
component from the master, Fig. 1b. This method of signal synthesis can be straightforwardly scaled further - e.g. two QPSK-like streams can be combined with subsequent carrier removal to obtain a 16 QAM signal (see Fig. 1c).

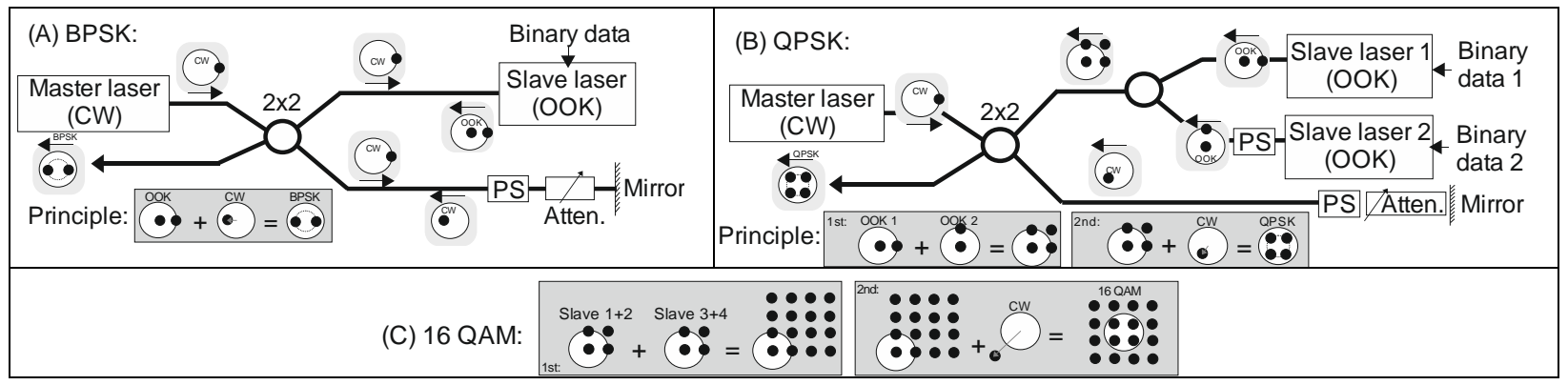

Fig. 1: Principle of operation ( ‘'’ means coherent addition using a coupler). PS: phase shifter.

\section{Optical injection locking and BPSK}

To validate the approach we built the setup shown in Fig 1a. For convenience, we used a tunable laser as the master. The slave laser was an Eblana discrete-mode laser optimized for $10 \mathrm{Gbit} / \mathrm{s}$ operation [6]. The laser was biased at 25 $\mathrm{mA}$ and the modulation depth was $1.2 \mathrm{~V}$ (p-p) (RF power of $4 \mathrm{dBm}$ ). First, we observed the slave laser output (by removing the reflective mirror in Fig. 1a) using a fast sampling oscilloscope. The eye diagrams are shown in Tab. 1. Here we first see that the directly-detected eye of the free-running slave laser (without IL) exhibits significant distortion (with considerable overshooting) as compared to the IL case for which the eye closely resembles that of the RF drive signal. The phase stability (residual chirp) was characterized by observing the modulated output after a $10-\mathrm{GHz}$ delayed line interferometer, DLI (Tab. $1,2^{\text {nd }}$ column), where we see a very significant improvement in eye opening for IL versus free-running operation of the slave laser. Following this experiment, we combined the slave laser output with a component of the master output (Fig. 1a). To stabilize the phase difference between the two signals (modulated slave and $\mathrm{CW}$ ), we monitored the output with a slow photodetector, processed the error signal with a feedback loop filter, and fed the (sub- $\mathrm{kHz}$ ) error-signal back to a PZT-based fibre stretcher (phase shifter, PS). The DLI-demodulated output together with a constellation diagram (obtained using a homodyne constellation analysis using 4096 points) is shown in Tab. 2. Here we see that signal is stable over long time scales as demonstrated with the sampling oscilloscope measurement that was acquired over a period of several seconds. We measured the BER of this signal and obtained error free $\left(B E R<10^{-10}\right)$ performance. The constellation shows two phase levels with close to straight transitions between them - similar to a BPSK signal generated using an external $\mathrm{LiNbO}_{3}$ modulator. The rms amplitude and phase magnitude errors, and error vector magnitude (EVM) were, $12 \%$, 7 degrees, and $18 \%$, respectively.

TAB 1: Modulated slave output, IL: Injection-locked.

\begin{tabular}{|l|c|c|}
\hline & Without IL & With IL \\
\hline Directly: & & \\
\hline After & & \\
10-GHz DLI: & & \\
\hline
\end{tabular}

TAB 2: Generated BPSK (slave combined with the CW master).

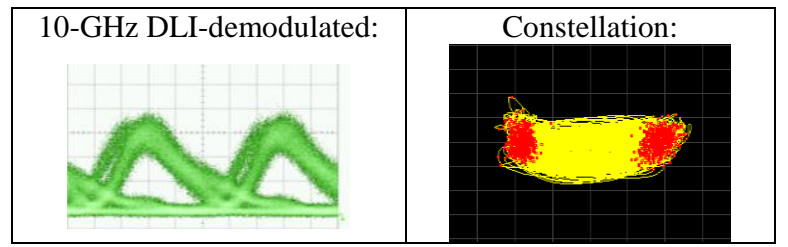

\section{QPSK}

The experimental setup for QPSK generation from two electrical data streams is shown in Fig. 2. The setup follows the basic scheme shown in Fig. 1b. The increased complexity as compared to Fig. $1 \mathrm{~b}$ is due to modifications incorporated to provide added flexibility, e.g., the possibility to control the injected optical power without compromising the output power (adding an attenuator in Fig. $1 \mathrm{~b}$ in front of the slave laser would reduce both the injected and emitted power). Between the master and the output, the light takes three different optical paths before being re-combined (CW, Slave 1\&2). To relax the requirement on the master linewidth, these three paths should be as closely balanced in length as possible - in our case $<20 \mathrm{~cm}$. Further, to minimize phase drifts due to environmental factors we kept the three fibres as close together as possible. As a result only sub-kHz bandwidth feedback was needed to stabilise the path difference as applied via two PZT fibre stretchers, see Fig. 2. The relevant spectral traces are shown in Fig. 3. First, we see that injection locking, as expected, reduces the chirp - substantially 
narrowing the spectral content of the modulated signal. The two OOK-modulated slaves combined together still have a carrier, which is removed by proper control of the power and phase of the added CW master component. Measurements with an optical resolution of $0.1 \mathrm{~nm}$ (not presented herein) showed the OSNR of the generated QPSK signal to be $32 \mathrm{~dB}$.

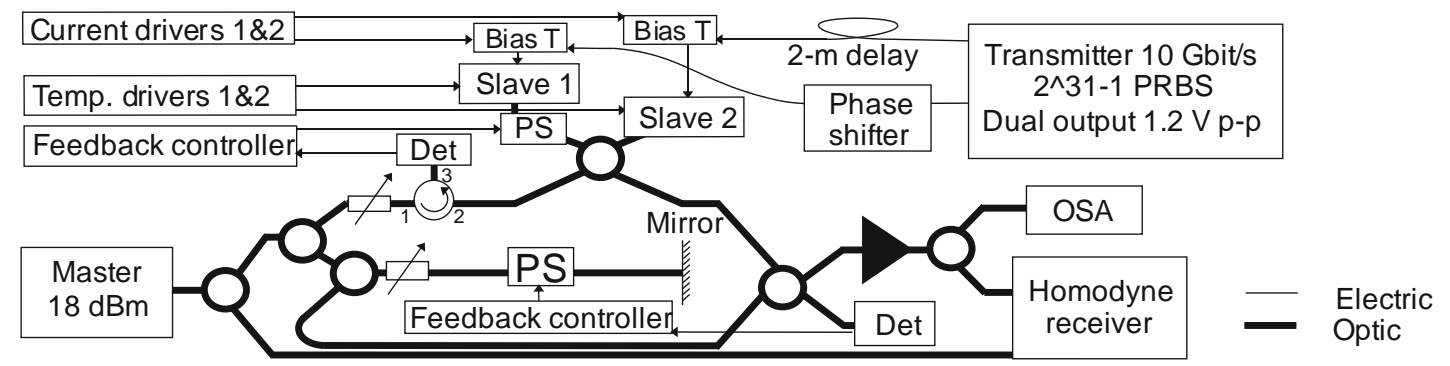

Fig. 2: Realized setup for QPSK modulation. PS: phase shifter.

Subsequently, we used a homodyne coherent receiver to perform constellation analysis and to measure the BER, see Fig. 4. The rms amplitude and phase magnitude errors, and EVM were 12\%, $7.5 \mathrm{deg}$, and 18\%, respectively. As for the BER, the signal was error-free as measured over time periods of several minutes, during which more than $10^{6}$ samples were acquired, confirming a BER below $10^{-6}$. The BER curve is measured by varying the optical power at the receiver and is shown in Fig. 4.

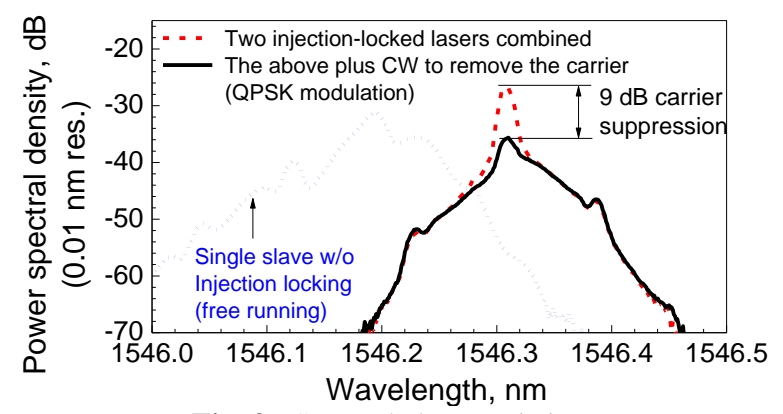

Fig. 3: Spectral characteristics.

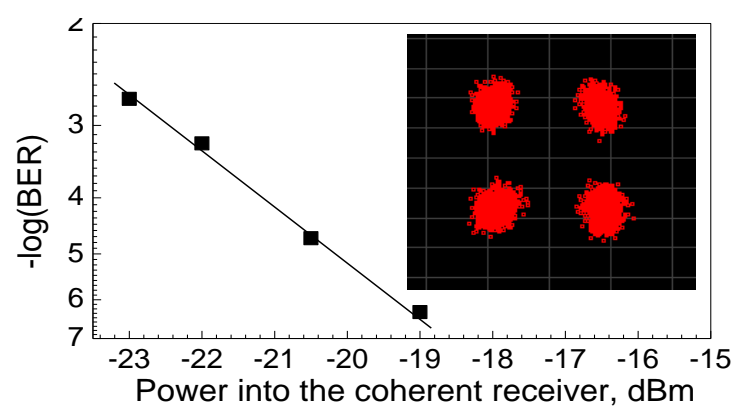

Fig. 4: BER and QPSK constellation (4096 points).

\section{Discussion and Conclusions}

The measured EVMs for QPSK and BPSK signals were similar, strongly suggesting that 16-QAM and higher formats should be achievable. Moreover, directly-modulated lasers are typically operated at limited extinction ratios (3-7 dB). Our method can suppress the chirp via injection locking and, at the same time, can improve the extinction ratio by destructively interfering the modulated signal with a small portion of the master laser, thereby mitigating two main limitations associated with the use of directly-modulated lasers for low cost OOK communications. Finally, injection locking can significantly enhance the laser modulation bandwidth - e.g., in [7] a 3-dB bandwidth of $80 \mathrm{GHz}$ was achieved. This would allow our scheme to operate at up to $160 \mathrm{Gbaud}$. Even without any bandwidth enhancement, 40 Gbaud operation should be achieveable, e.g., by using the lasers reported in [8].

Synthesis of BPSK and QPSK at 10 Gbaud using directly-modulated, injection-locked semiconductor lasers is demonstrated, with error-free operation obtained. This potentially low-cost scheme should be readily extended to higher modulation formats and speeds and is fully amenable to photonic integration.

\section{References}

[1] A. Chiba et al, "16-level QAM by monolithic quad-parallel Mach-Zehnder optical modulator," Electron. Lett. 46, 220 (2010).

[2] K.Y. Cho et al, "10-Gb/s, 80-km Reach RSOA-Based WDM PON Employing QPSK Signal and Sel-Homodyne Receiver," in OFC 2012, OW1B.

[3] W. Jia et al, "Generation of 20-Gb/s RZ-DQPSK Signal using a Directly Modulated Chirp Managed Laser," in OFC 2011, OThE4.

[4] R. Hui, "Optical PSK Modulation Using Injection-Locked DFB Semiconductor Lasers," Photon. Tech. Lett. 2, 743 (1990).

[5] S. Mohrdiek, H. Burkhard, and H. Walter, "Chirp Reduction of Directly Modulated Semiconductor Lasers at 10 Gb/s by Strong CW Light Injection, "J. Lightwave Technol. 12, 418 (1994).

[6] R. Phelan et al, "- $40^{\circ} \mathrm{C}<\mathrm{T}<95^{\circ} \mathrm{C}$ mode-hop-free operation of uncooled AlGaInAs-MQW discrete-mode laser diode with emission at $\lambda=1.3$ $\mu \mathrm{m}$, "Electron. Lett. 45, 43 (2009).

[7] E.K. Lau et al, "Strong optical injection-locked semiconductor laser demonstrating >100-GHz resonance frequencies and 80-GHz intrinsic bandwidths," Opt. Express 16, 6609 (2008).

[8] A. Uetake et al, "40-Gbps Direct Modulation of $1.55 \mu \mathrm{m}$ AlGaInAs Semi-Insulationg Buried-Heterostructure Distributed Reflector Lasers up to $85^{\circ} \mathrm{C}$," in LEOS Meeting 2009, paper ThBB. 